\title{
Psychological Impact of Covid-19 Pandemic on the Healthcare Workers of a Tertiary Care Hospital of Lahore
}

\author{
Maria Aslam', Sahibzada M. Ahmed Kharral'2, Farooq Azam Khan ${ }^{3}$
}

Professor, Department of Pathology, Sharif Medical \& Dental College, Lahore Pakistan
Data collection, Manuscript writing
2 Forrest Family Practice; Bunbury; Western Australia
Literature review, Statistical analysis

3 Associate Professor, Department of Orthopedics, Sharif Medical \& Dental College, Lahore Pakistan 3 Article layout, References layout

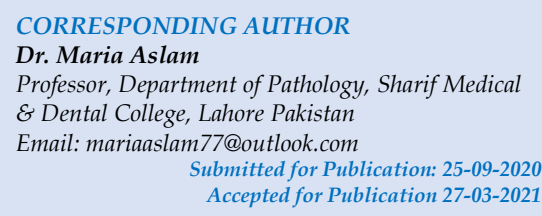
Accepted for Publication 27-03-2021

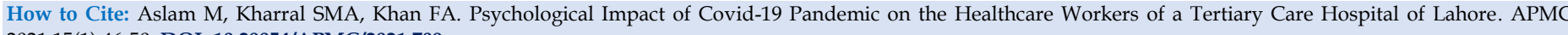
2021;15(1):46-50. DOI: 10.29054/APMC/2021.709

\section{ABSTRACT}

Background: The psychological stress associated with the COVID-19 pandemic can have long-term effects on the health and coping abilities of HCWs. COVID 19 has exhausted the healthcare workers globally both physically and mentally. This article focuses on the Psychological stresses faced by the healthcare workers of a tertiary setup. Objective: To determine the psychological impact of the COVID-19 pandemic on the healthcare workers (HCWs) of a tertiary care hospital of Lahore. Study Design: Descriptive, cross-sectional study. Settings: Sharif Medical City Hospital, Lahore Pakistan. Duration: From July 2020 to August 2020. Methodology: A total of 82 healthcare workers were enrolled in the study on a voluntary basis by convenient sampling technique and filled an online questionnaire including demographic information, informed consent, validated Depression, Anxiety and Stress Scales (DASS-21) and the factors causing psychological distress. The data was analyzed using the Statistical Package for the Social Sciences (SPSS) version 24. Results: Out of 82 HCWs, 47(57.3\%) had stress, 42(51.2\%) had depression and 29(35.4\%) had anxiety. Most of the HCWs with stress had mild and moderate stress. The majority of the HCWs were suffering from moderate depression and moderate anxiety. The most common factor causing psychological stress among HCWs was the fear of getting infected with COVID-19 followed by chances of spreading the disease to family members and the non-availability of personal protective equipment. Conclusion: The COVID-19 pandemic has a great psychological impact on the healthcare workers in Pakistan. A large proportion of HCWs suffered from stress $(57.3 \%)$, depression $(51.2 \%)$ and anxiety $(35.4 \%)$. The most common factors causing psychological distress in HCWs were the fear of getting infected with COVID-19 (80.5\%), chances of spreading the disease to family members $(65.9 \%)$ and the non-availability of personal protective equipment $(59.8 \%)$.

Keywords: COVID-19, Pandemic, Healthcare workers, Psychological impact.

\section{INTRODUCTION}

Coronavirus disease has emerged as a global pandemic near the end of 2019 that has spread rapidly since its identification. ${ }^{1,2}$ It is a leading cause of morbidity, mortality and financial costs. ${ }^{3}$ About 5165481 cases of COVID-19 had been reported with 336430 deaths worldwide to the World Health Organization (WHO) by $24^{\text {th }}$ May 2020. ${ }^{4}$ The disease has affected greater than 200 countries across the globe and has a fatality rate of $7 \% .{ }^{5}$ The WHO reported that $14 \%$ of the patients with COVID19 develop severe disease requiring hospitalization, $5 \%$ of cases have very severe disease with the need for intensive care therapy \& ventilation and $4 \%$ of the patients die from the disease. The mortality rate of COVID-19 is $4 \%$ in China, $10 \%$ in the rest of Asia, $4 \%$ in Europe and $10 \%$ in America. The infection rate in Asia reached up to $80 \% .{ }^{3}$ In the past, two outbreaks of Coronaviruses occurred, one with severe acute respiratory syndrome (SARS)-CoV and the other with the Middle East respiratory syndrome (MERS)-CoV. ${ }^{6}$
Several individuals presented with pneumonia of idiopathic cause in December 2019 in Wuhan, China which attracted national and global attention. A novel coronavirus (SARS-CoV-2, also called 2019-nCoV) was isolated from these patients in January 2020 by polymerase chain reaction (PCR) and next-generation sequencing. On January 30, 2020, WHO announced COVID-19 as an epidemic and Public Health Emergency of International Concern (PHEIC). Till February 2020, 80,239 patients with Coronavirus were confirmed globally leading to 2700 deaths. ${ }^{7}$

Person to person transmission occurs by respiratory droplets and fomites. In the majority of the cases, the virus is transmitted from symptomatic patients but transmission can also occur during the asymptomatic incubation period. ${ }^{8}$ Coronaviruses are enveloped, RNA viruses with a diameter of $60-140 \mathrm{~nm}$. The virus has a crown-like appearance when seen in the electron microscope due to spike-like projections protruding from it. ${ }^{2}$ The clinical picture of COVID-19 varies causing 
asymptomatic infection or acute respiratory distress syndrome and multiorgan dysfunction. The most common clinical features are fever, cough, headache, sore throat, shortness of breath, fatigue, myalgia and conjunctivitis. During disease progression, patients can develop pneumonia, acute respiratory distress syndrome, respiratory failure, shock, acute kidney injury and death. The mortality rate is $4 \%-11 \%$ in hospitalized patients. ${ }^{1}$ There is no drug or vaccine available yet for treating COVID-19. Several trials are under process but it will take months or even years to develop a treatment regime or vaccine. $^{2}$

Braquehais et al. reported various factors during the COVID-19 pandemic that are responsible for psychological stress among healthcare workers (HCWs). These factors are the high mortality and morbidity associated with the disease, lack of effective treatment/vaccine for COVID-19, non-availability of personal protective equipment (PPE), fear of being getting the disease and infecting their family members. Many of the HCWs also got infected and quarantined during the pandemic or even required hospitalization. The stress level was even higher in these HCWs. ${ }^{4}$

Literature has reported the evidence of significant stress, anxiety and depression among the HCWs in the past epidemics of Coronavirus by SARS and MERS. The COVID-19 pandemic has an unexpected psychological impact on the HCWs especially in countries that had not experienced past Coronavirus epidemics. The situation worsened with reduced availability of resources and workforce capacity in the hospitals. As more and more HCWs became infected and were quarantined, the workload on the HCWs increased. The psychological stress associated with the COVID-19 pandemic can have long-term effects on the health and coping abilities of HCWs. This study was designed to determine the impact of COVID-19 disease on HCWs in terms of psychological stress, anxiety \& depression and the factors causing psychological distress. It will help us in developing ways to negate the psychological impact and promote the psychological wellbeing of HCWs. The provision of psychological support to the HCWs during the pandemic is one of the most difficult challenges in mental health. It will also help us to cope with future epidemics/pandemics.

\section{METHODOLOGY}

Study Design: Descriptive, cross-sectional study

Settings: Sharif Medical City Hospital, Lahore

Duration: From July 2020 to August 2020

Sample Size: Eighty-two healthcare workers.

Sampling Technique: Convenient sampling technique

Data Collection Procedure: After approval from the ethical committee of the institution, the HCWs were enrolled in the study. The participation was entirely on a voluntary basis and the HCWs filled an online questionnaire including demographic information, informed consent, validated Depression, Anxiety and Stress Scale (DASS-21) and the factors causing psychological distress. There are 21 statements in the scale with 7 items each of depression, anxiety and stress scale. The score of each statement ranges from 0 to 3 . The interpretation of scores is as follows: 0 - Never, 1 Sometimes, 2 - Often and 3 - Almost always. The cut-off scores to label normal, mild, moderate, severe and extremely severe depression, anxiety and stress are shown in table $1 .^{10}$

Table 1: Severity of Depression, Anxiety and Stress Based on the DASS-21 Scale

\begin{tabular}{|c|c|c|c|}
\hline & Depression & Anxiety & Stress \\
\hline Normal & $0-9$ & $0-7$ & $0-14$ \\
\hline Mild & $10-13$ & $8-9$ & $15-18$ \\
\hline Moderate & $14-20$ & $10-14$ & $19-25$ \\
\hline Severe & $21-27$ & $15-19$ & $26-33$ \\
\hline Extremely Severe & $28+$ & $20+$ & $34+$ \\
\hline${ }^{*}$ The final score will be calculated by multiplying the obtained score by 2.
\end{tabular}

The data was analyzed using the Statistical Package for the Social Sciences (SPSS) version 24. The qualitative variables such as gender, type of healthcare professional and the severity of DASS-21 were described using frequency and percentage. The quantitative variables such as age, depression, anxiety and stress scores were expressed using mean and standard deviation. Chisquare statistic was used to determine the association of depression, anxiety and stress scores with the age, gender and type of healthcare professional. A p-value of $\leq 0.05$ was statistically significant.

\section{RESULTS}

The mean age of the HCWs was $41.41 \pm 4.84$ years. The minimum age was 34 years and the maximum age was 54 years. Out of 82 participants, 51(62.2\%) were males and $31(37.8 \%)$ were females. Fifty-six $(68.3 \%)$ HCWs were doctors, $16(19.5 \%)$ were nurses and 10(12.2\%) were allied health professionals.

The mean stress score was $15.90 \pm 7.22$ with the lowest score of 2 and the highest score of 38 . Thirty-five (42.7\%) HCWs had a normal stress score whereas 47(57.3\%) HCWs had stress. Out of these 47 HCWs, 20(24.4\%) had mild stress, $20(24.4 \%)$ had moderate stress, $5(6.1 \%)$ had severe stress and $2(2.4 \%)$ had extremely severe stress.

The mean depression score was $10.15 \pm 6.50$. The lowest score was 0 and the highest score was 24 . Out of 82 participants, $40(48.8 \%)$ had a normal depression score whereas $42(51.2 \%)$ were suffering from depression. Fourteen $(17.1 \%)$ participants had mild depression, $23(28 \%)$ had moderate depression and $5(6.1 \%)$ had severe depression. 
The mean anxiety score was $5.93 \pm 4.99$. The minimum score was 0 and the maximum score was 24 . Fifty-three $(64.6 \%) \mathrm{HCWs}$ had a normal anxiety score and the scores of $29(35.4 \%)$ HCWs were suggestive of anxiety. Out of these $29 \mathrm{HCWs}, 10(12.2 \%), 14(17.1 \%), 4(4.9 \%)$ and $1(1.2 \%)$ HCWs had mild, moderate, severe and extremely severe anxiety, respectively. Table 2 and Figure 1 show the stress, depression and anxiety scores of the study subject.

Table 2: Stress, Depression and Anxiety Scores of the Study Subjects

\begin{tabular}{|c|c|c|c|}
\hline Score & Stress & Depression & Anxiety \\
\hline Normal & $35(42.7 \%)$ & $40(48.8 \%)$ & $53(64.6 \%)$ \\
\hline Mild & $20(24.4 \%)$ & $14(17.1 \%)$ & $10(12.2 \%)$ \\
\hline Moderate & $20(24.4 \%)$ & $23(28 \%)$ & $14(17.1 \%)$ \\
\hline Severe & $5(6.1 \%)$ & $5(6.1 \%)$ & $4(4.9 \%)$ \\
\hline Extremely Severe & $2(2.4 \%)$ & $0(0 \%)$ & $1(1.2 \%)$ \\
\hline
\end{tabular}

Figure 1: Stress, Depression and Anxiety Scores of the Study Subjects

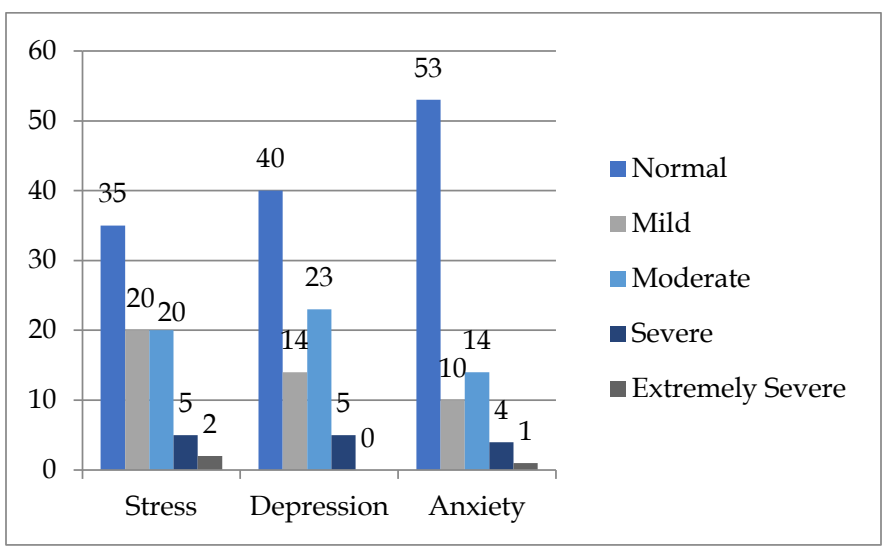

There was a statistically insignificant association between the age \& the type of healthcare professional and the stress score ( $p$-value $=0.159$ and 0.296 , respectively). However, the stress score was significantly higher in females than in males $(p$-value $=0.001)$. The depression score of the HCWs had an insignificant relationship with their age, gender and type with the $p$-value of $0.296,0.735$ and 0.363 , respectively. The association between the age of HCWs and anxiety was significant ( $p$-value $=0.000)$. However, the association was insignificant between age $\&$ type of healthcare professional and the anxiety score ( $\mathrm{p}$ value $=0.490$ and 0.924 , respectively) .

The most common factor leading to psychological distress in HCWs was the fear of getting infected with COVID-19 (80.5\%). Other factors were the chances of spreading the disease to their family members (65.9\%) and the non-availability of personal protective equipment $(59.8 \%)$. These factors are shown in table 3.
Table 3: Factors Leading to Psychological Distress in Healthcare Workers

\begin{tabular}{|l|c|}
\hline Factors Leading to Psychological Distress & $\begin{array}{c}\text { Frequency } \\
\text { (Percentage) }\end{array}$ \\
\hline Fear of getting infected with COVID-19 & $66(80.5 \%)$ \\
\hline $\begin{array}{l}\text { Chances of spreading the disease to } \\
\text { family members }\end{array}$ & $54(65.9 \%)$ \\
\hline $\begin{array}{l}\text { Non-availability of personal protective } \\
\text { equipment }\end{array}$ & $49(59.8 \%)$ \\
\hline $\begin{array}{l}\text { Lack of security \& administrative issues } \\
\text { in hospitals }\end{array}$ & $40(48.8 \%)$ \\
\hline $\begin{array}{l}\text { Increasing number of COVID-19 cases } \\
\text { day by day }\end{array}$ & $38(46.3 \%)$ \\
\hline $\begin{array}{l}\text { Lack of awareness among patients \& their } \\
\text { attendants }\end{array}$ & $29(35.4 \%)$ \\
\hline Increased workload & $10(12.2 \%)$ \\
\hline
\end{tabular}

\section{DISCUSSION}

Coronavirus disease is the biggest challenge for healthcare systems and their workers. The COVID-19 pandemic has overburdened healthcare systems all over the world. Healthcare workers are not only at the frontline risk of developing the disease but are also vulnerable to negative psychological outcomes. A systematic review conducted on the mental health of HCWs during the COVID-19 pandemic revealed that the psychological stress ranges from $7 \%$ to $97 \%$ (median $37 \%$ ), anxiety ranges from $9 \%$ to $90 \%$ (median $24 \%$ ) and depression ranges from $5 \%$ to $51 \%$ (median $21 \%$ ) in HCWs. ${ }^{11}$

The mean age of the HCWs was 41.41 years in our study. The mean age was 31 years in a study by Tan et al. and 29 years by Chew et al. ${ }^{12,13}$ Our results showed that the majority $(62.2 \%)$ of the HCWs who participated in our study were males and $37.8 \%$ were females. Sandesh et al. reported that $57.1 \%$ of the HCWs were males and $42.9 \%$ were females in his study. ${ }^{14}$ In contrast, in a study by Tan et al. conducted in Singapore, $68.3 \%$ of the HCWs were females and $31.7 \%$ were males. ${ }^{12}$ In another study, $64.3 \%$ of the HCWs were females and $35.7 \%$ were males. ${ }^{13}$

In our study, most of the HCWs were doctors followed by nurses and allied healthcare professionals. In another study, there were $34.3 \%$ nurses, $28.7 \%$ doctors, $13.8 \%$ allied healthcare workers, $7.7 \%$ maintenance workers, $7 \%$ administrative staff, $6.4 \%$ clerical staff and $2.1 \%$ technicians. ${ }^{12}$ The majority of the HCWs were nurses $(39.2 \%)$ in a study followed by physicians $(29.6 \%)$ and allied health care professionals $(10.6 \%)$. In addition, there were $4.4 \%$ of technicians, $6.2 \%$ clerical staff, $4.3 \%$ administrator staff and $5.7 \%$ maintenance staff. ${ }^{13}$

In our study, the mean scores of stress, depression and anxiety were $15.90 \pm 7.22,10.15 \pm 6.50$ and $5.93 \pm 4.99$, respectively. Another study reported even higher scores; the mean stress score of $20.12 \pm 12.0$, depression score of $18.12 \pm 10$ and anxiety score of $19.01 \pm 9.2 .^{14}$ In contrast, the 
mean scores were low in another study. The mean score of stress was $4.62 \pm 5.54$, depression was $3.08 \pm 4.86$ and anxiety was $3.21 \pm 4.29 .13$

Our results showed that $57.3 \%$ HCWs had stress, $51.2 \%$ were suffering from depression and 35.4\% HCWs had anxiety. In the study by Sandesh et al. conducted in Karachi, Pakistan, there were a total of 112 participants. Out of these, $90.2 \%$ HCWs had depression, $95.5 \%$ had anxiety and $96.4 \%$ had stress. ${ }^{14}$ In China, a high percentage of depression $(50 \%)$, anxiety $(45 \%)$ and distress $(72 \%)$ were reported in HCWs. But most of them had a mild score..$^{15}$ The scores were low in a study done in Singapore. Out of 470 participants, $68(14.5 \%)$ HCWs had anxiety, $42(8.9 \%)$ had depression and $31(6.6 \%)$ had stress. ${ }^{12}$ In another study, anxiety was present in $15.7 \%$ HCWs, depression in 10.6\% HCWs and stress in 5.2\% HCWs in Singapore and India. ${ }^{13}$ According to a systematic review, out of $230 \mathrm{HCWs}, 53(23.04 \%)$ had psychological issues. Severe, moderate and mild anxiety was present in $2.17 \%, 4.78 \%$ and $16.09 \%$ of the HCWs. Stress was present in $27.4 \%$ and depression in $50.4 \%$ of the HCWs. ${ }^{16}$

In our study, most of the HCWs with stress had mild and moderate stress. The majority of the HCWs were suffering from moderate depression and moderate anxiety. In contrast, in another study, most of the HCWs had moderate and severe depression, stress and anxiety. ${ }^{13}$ Similarly, in another study, $72.3 \%$ of the HCWs had moderate to extremely severe depression, $85.7 \%$ HCWs had moderate to extremely severe anxiety and 90.1\% HCWs had moderate to very severe stress. ${ }^{14}$

In our study, only the stress score was significantly higher in females than in males. There was no significant association between the type of healthcare professional and the stress, depression \& anxiety score. Other studies reported that female HCWs and nurses had higher scores as compared to males and other healthcare professionals. 15,16

In our study, the most common factor leading to psychological distress in HCWs was the fear of getting infected with COVID-19 (80.5\%) followed by the chances of spreading the disease to their family members $(65.9 \%)$ and the non-availability of personal protective equipment $(59.8 \%)$. Another study reported that the most common factors were the risk of transmitting the disease to family members $(89.2 \%)$, fear of getting infected $(80.3 \%)$, increased workload $(64.2 \%)$, non-availability of PPE $(62.5 \%)$, lack of security $(62.5 \%)$ and unawareness of the people about COVID-19 (46.4\%). ${ }^{14}$

\section{CONCLUSION}

The COVID-19 pandemic has a great psychological impact on the healthcare workers in Pakistan. A large proportion of HCWs suffered from stress (57.3\%), depression (51.2\%) and anxiety (35.4\%). The most common factors causing psychological distress in HCWs were the fear of getting infected with COVID-19, the chances of spreading the disease to family members and the non-availability of personal protective equipment.

\section{LIMITATIONS}

The study should be conducted on a large number of healthcare workers from different institutions.

\section{SUGGESTIONS / RECOMMENDATIONS}

- The preventable factors causing psychological distress in HCWs such as the non-availability of PPE, lack of security \& administrative issues in hospitals should be solved.

- The duty hour schedule of the HCWs should be made so that it does not exceed their working capacity.

- The HCWs especially those directly dealing with the COVID-19 positive patients should be encouraged for their work.

- Psychological support teams should be made in hospitals to evaluate the psychological status of HCWs and provide them continuous support.

\section{CONFLICT OF INTEREST / DISCLOSURE}

There is no conflict of interest of authors in the study.

\section{ACKNOWLEDGEMENTS}

None.

\section{REFERENCES}

1. Singhal T. A review of Coronavirus disease - 2019 (COVID-19). Indian J Pediatr. 2020;87(4):281-6.

2. Li G, De Clercq E. Therapeutic options for the 2019 novel Coronavirus (2019-nCoV). Nat Rev Drug Discov. 2020;19(3):149-50.

3. Grech V. Unknown unknowns - COVID-19 and potential global mortality. Early Hum Dev. 2020;44:105026.

4. Braquehais MD, Vargas-Caceres C, Gomez-Duran E, Nieva G, Valero S, Casas M, et al. The impact of the COVID-19 pandemic on the mental health of healthcare professionals. QJM- Int J Med. 2020;113(9):613-7.

5. Felice C, Di Tanna GC, Zanus G, Grossi U. Impact of COVID-19 outbreak on healthcare workers in Italy: results from a national Esurvey. J Community Health. 2020;45(4):675-83.

6. Rothan HA, Byrareddy SN. The epidemiology and pathogenesis of Coronavirus disease (COVID-19) outbreak. J Autoimmun. 2020;109:102433.

7. Jiang F, Deng L, Zhang L, Cai Y, Cheung CW, Xia Z. Review of the clinical characteristics of Coronavirus disease 2019 (COVID-19). J Gen Intern Med. 2020;35(5):1545-9.

8. Sohrabia C, Alsafib Z, O'Neilla N, Khanb M, Kerwanc A, Al-Jabirc A, et al. World Health Organization declares global emergency: a review of the 2019 novel Coronavirus (COVID-19). Int J Surg. 2020;76:71-6.

9. Silva RG, Figueiredo-Braga M. Evaluation of the relationships among happiness, stress, anxiety, and depression in pharmacy students. Curr Pharm Teach Learn. 2018;10(7):903-10.

10. Arab M, Rafiei H, Safarizadeh MH, Ahmadi JS, Safarizadeh MM. Stress, anxiety and depression among medical university students and its relationship with their level of happiness. IOSR JNHS. 2016;5(1):44-7.

11. Muller AE, Hafstad EV, Himmels JPW, Smedslund G, Flottorp S, Stensland SO, et al. The mental health impact of the Covid-19 
pandemic on healthcare workers and interventions to help them: a rapid systematic review. Psychiatry Res. 2020;293:113441.

12. Tan BYQ, Chew NWS, Lee GKH, Jing M, Goh Y, Yeo LLL, et al. Psychological impact of the COVID-19 pandemic on healthcare workers in Singapore. Ann Intern Med. 2020;173(4):317-20.

13. Chew NWS, Lee GKH, Tan BYQ, Jing M, Goh Y, Ngiama NJH, et al. A multinational, multicentre study on the psychological outcomes and associated physical symptoms amongst healthcare workers during COVID-19 outbreak. Brain Behav Immun. 2020;88:559-65.
14. Sandesh R, Shahid W, Dev K, Mandhan N, Shankar P, Shaikh A, et al. Impact of COVID-19 on the mental health of healthcare professionals in Pakistan. Cureus. 2020;12(7):e8974.

15. Lai J, Ma S, Wang Y, Cai Z, Hu J, Wei N, et al. Factors associated with mental health outcomes among healthcare workers exposed to Coronavirus disease 2019. JAMA Netw Open. 2020;3(3):e203976.

16. Shaukat N, Ali DM, Razzak J. Physical and mental health impacts of COVID-19 on healthcare workers: a scoping review. Int J Emerg Med. 2020;13:40-8. 